Bangladesh J. Sci. Ind. Res. 42(2), 203-212, 2007

\title{
Studies on Transition Temperature of the Superconducting Materials Related to Elastic Constants
}

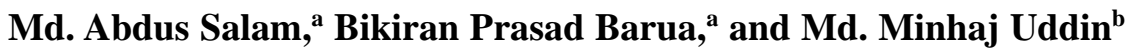 \\ ${ }^{a}$ BCSIR Laboratories, Chittagong and ${ }^{b}$ Department of Physics, \\ University of Chittagong, Bangladesh
}

\begin{abstract}
For a face-centered-cubic structure crystal the parameters like atomic mass $\mathrm{M}$, atomic number $\mathrm{Z}$, lattice spacing are the important factors in the determination of a many body Hamiltonian $\mathrm{H}$. Instead of using fcc lattice spacing we can also utilize atomic volume $\Omega$.Using the superconducting transition temperature expressed as $T_{c}=T_{c}{ }^{(M)}(Z, \Omega)$, a well defined region in superconducting elements is possible. $T_{c}$ may also be related to BCS theory through Debye temperature. This Debye temperature is related to elastic constants $C_{11}, C_{12}$ and $C_{44}$, atomic weight and atomic volume. Also $T_{c}$ for five fcc transitions metals is linear in the Cauchy deviation $C^{*}=\left(C_{12}-C_{44}\right) /\left(C_{12}+C_{44}\right)$, such linearity is also seen in bcc transition elements. Finally via elastic constants mass density and atomic volume, a correlation between $\mathrm{C}^{*}$ and the Debye $\Omega$ temperature has been established for the five fcc transition elements. This correlation indirectly sets up a relation between transition temperature and elastic constant.
\end{abstract}

\section{Introduction}

For heavy fermions superconductor, the superconducting transition temperatures are shown $T_{c}$ to correlate with a character istic energy $h^{2} /\left(m^{*} \xi^{2}\right), m^{*}$ being the effec tive mass and $\hat{\imath}$ the coherence length. It is very difficult to identify the correlations between superconducting properties and elastic properties of fcc elements due to their complex crystallographic structure. These correlations would be helpful to study the new classes of superconductors with relevant variables (Angilella, et al., 2000; Angilella, et al., 2002 and Angilella, et al. 2004).
According to the BCS theory (Schrieffer, 1964) the attraction caused by even a weak lattice distortion can overcome $\mathrm{h}^{2} /\left(\mathrm{m}^{*} \xi^{2}\right)$, $m^{*}$ strong Coulomb's repulsion. Thus the net effect is the attraction of two electrons via a lattice distortion to form a pair of electron known as the cooper pair, questions have come up regarding the role of strong electron-electron interactions in both the high $T_{c}$ cuprites and heavy fermions materials.

If we were able to solve the many-body Schrödinger equation for the superconduct- 
ing elements, then by treating the motion of nuclei and $T_{c}$ electrons on the same basement, plus full inclusion of electronelectron interactions. These interactions, such uncertainties engaged in separating electron lattice and Coulomb repulsions between electrons would be bypassed.

Let us consider some superconducting metallic elements. We treat these elements by the following procedure: Firstly we single-out the face-centered-cubic (fcc) lattice. We would need the atomic volume $\Omega$ (of course, essentially equivalently the lattice parameter a) to specify the structure. Again atomic number $\mathrm{Z}$ is required to signify the external potential which created by the nuclei. Any element that has a many-body Hamiltonian containing both electron and nuclear kinetic energy also need nuclear mass $\mathrm{M}$. We also take here some fundamental constants such as nuclear mass $h, e$ and electronic mass $m$.

For our structure which we have taken fcc, the superconducting transition temperature $T_{c}$ depends on $M, Z$ and $\Omega$.This is apart from the many-body partition function; once the Schrödinger equation has been solved from the fundamental constants such as Plank's constant $h, e$ the charge of electron and electronic mass $m$.

$$
T_{c}=T_{c}^{(\mathrm{M})}(\mathrm{Z}, \Omega)
$$

We have more than one atomic number for all other types of superconductors.

\section{Materials and Methods}

As described in the introduction in the beginning, the methodology of the work is given below. In section 1 described below we have represented $T_{c}=T_{c}^{(\mathrm{M})}(\mathrm{Z}, \Omega)$ to lead our present work. This is the work of testing that $T_{c}$ depends on atomic number $Z$ and atomic volume $\Omega$ in fcc transition elements. Our chosen five fcc elements are $\mathrm{Al}, \mathrm{Pb}, \mathrm{La}$, Ir and Th.This elements are considered in the $(Z, \Omega)$ plane with respect to their transition temperature. There is weak but relatively unimportant dependence of $T_{c}$ on nuclear isotopic mass. In section 2 we have introduced new quantity, Cauchy discrepancy which is related to $T_{c}$ i.e. the departure of $C_{12}$ from $C_{44}$ where these are two of the elastic constants ( $C_{11}$ being the other) required to characterize a cubic crystal. In section 3 using the essential ingredient of BCS theory the Cauchy deviation relates to the Debye temperature. In section 4 we have summarized and designed some clue for advance research both theoretical and experimental. At last we have shown in the Appendix for that a particular critical field $H_{c}(0)$ and energy gap $E_{g}(0)$ can be des-cribed as function of the Cauchy discrepancy. This function considers zero temperature properties.

Evidence of correlation between transition temperatures $T_{c}$ and atomic number $(z)$ or atomic volume $(\Omega)$ in fcc transition elements.

For graphical view we have made a plot for the five fcc elements in the $(\Omega, \mathrm{Z})$ plane and 
attached every experimental values of $T_{c}$ to the recognized coordinates.

The functional form $T_{c}(\Omega, \mathrm{Z})$ comments us that (1) transition temperature significantly decreases with increasing atomic number for constant volume (2) For constant Z, there is plainly substantial variation of $T_{c}$ with atomic volume, which is proportional to the reciprocal of the concentration, It may be mentioned here that atomic volume $\Omega$ and atomic number $\mathrm{Z}$ are important variables in characterizing $T_{c}$ for a definite element. It is also observed that high pressure can turn many elements into superconductors via an insulator-metal transition.

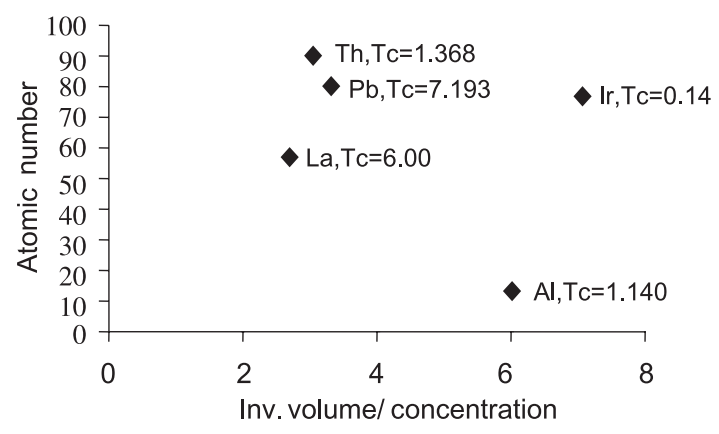

Fig.1. Shows five superconducting fcc transition elements in the $(\Omega, Z)$ plane. Actual abscissa is the reciprocal of the atomic volume $\Omega^{-1}$. To each point the value of the critical temperature $T_{c}$ is shown.

Transition $T_{c}$ temperature usually increases with increasing pressure for most super - conducting elements at ambient pressure (See Table I). Within BCS theory [see also Eq. below] or its extension by McMillan, this is usually justified in terms of a pressureinduced lattice stiffening which reduces the electron-phonon constant at a more rapid rate than the electron density of states at the Fermi level is increased (Hochheimer, et al. 2001).

Table I. Presssure derivatives of $T_{c}{ }^{5}$,experimental bulk moduli ${ }^{6}$, and inferred partial derivatives of $T_{c}$ with respect to $\Omega$ at constant Z, Eq.(2), of the fcc superconducting transition metals at $P=0$.

\begin{tabular}{l|l|l|l|l|l}
\hline Elements & $\mathrm{Al}$ & $\mathrm{Th}$ & $\mathrm{Pb}$ & $\mathrm{Ir}$ & $\mathrm{La}$ \\
\hline$\frac{\partial T_{c}}{\partial P}[\mathrm{~K} / \mathrm{GPa}]$ & 1.15 & 7.09 & 276.42 & -39.65 & 445.19 \\
$B[\mathrm{GPa}]$ & 72.2 & 54.3 & 43.00 & 355.00 & 24.30 \\
$\frac{\partial T_{c}}{\partial \Omega}\left[10^{31} \mathrm{~K} \cdot \mathrm{m}^{-3}\right]$ & -0.50 & -1.17 & -39.22 & 0.99 & -29.21 \\
\hline
\end{tabular}

Pressure derivatives of $T_{c}$ can then are straightforwardly related to volume derivatives at constant $\mathrm{Z}$ from the relation.

$\frac{\partial \log T_{c}}{\partial \log \Omega}=-B \frac{\partial \log T_{c}}{\partial P}$

Where B is the bulk modulus (See Table I)

However, this partial derivative indicates us that $T_{c}$ depend apparently in a sensitive way on these two variables. Because, chosen fcc structure allows the detailed form of the function $T_{c}(\Omega, \mathrm{Z})$ for this structure. We know that there is a correlation between $T_{c}$ and the Cauchydiscrepancy $C^{*}=\left(C_{12}-C_{44}\right) /\left(C_{12}+\right.$ $C_{44}$ ) between elastic constants. By applying this clue we can characterize $T_{c}$ by a single variable, rather than the pair $(\Omega, \mathrm{Z})$ used in fig. 1. 


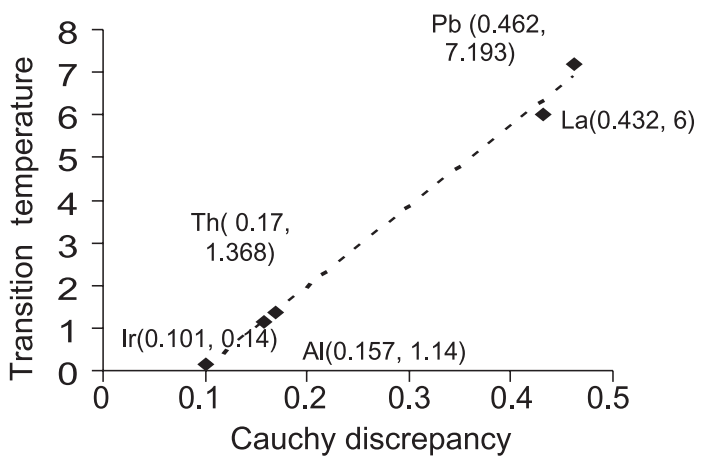

Fig.2. Superconducting transition temperatures for fcc transition elements correlated with Cauchy discrepancy, Eq(3). Solid line is best fit to the point [see text for discursion, in particular eq.(4)]. Redrawn from ref ${ }^{8}$ see also ${ }^{6}$ for an update on elastic constants data.

Correlation between superconducting transition temperature $T_{c}$ and the cauchy discrepancy $C^{*}$ in face-centered-cubic transition metals

For face-centered-cubic transition metals, an empirical relationship exists between the superconducting transition temperature and Cauchy discrepancy. This correlation may arise from many-atom effects.

We have drawn a pot of verses Cauchy discrepancy for five fcc elements by following the previous work of H.M Ledbetter (Myers, 1997) carried out almost a quarter of Century ago. The quantity defined by

$$
C^{*}=\frac{C_{12}-C_{44}}{C_{12}+C_{44}}
$$

Ledbetter (Myers, 1997) also included some alloys, namely $N b_{0.9} Z r_{0.1}, N b_{0.4} T i_{0.6}$ and $\mathrm{Ti}_{0.7} v_{0.3}$ but we have omitted these from redrawn fig. 2. It should be mentioned, that a high pressure, nonmagnetic, but also a non bcc phase of iron has been recently reported to display superconductivity with $T_{c}<2 k$ between 15 and $30 \mathrm{GPa}$. (Shimizu, et al., 2001) The equation of the straight line drawn in fig. 2 is

$$
T_{c}[\mathrm{~K}]=A C^{*}-\mathrm{B}
$$

Here $A=17.7 \mathrm{~K}$ and $\mathrm{B}=1.65 \mathrm{~K}$, we have no relevant theory to evaluate A\&B. Several previous studies relate elastic constants to superconductivity. The physical meaning of equation (4) indicates us to think the relation with $C^{*}$ to a basic ingredient of BCS theory, the Debye temperature $\theta_{\mathrm{D}}$.

Correlation between cauchy discrepancy $C^{*}$ and debye temperature, $\theta_{D}$ which give the scale of $T_{c}$ in the bcs theory

The criterion for the transition temperature of an element or alloy involves the density of states $N\left(E_{F}\right)$ at the Fermi level and the electron-phonon coupling constant $\mathrm{V}$ which can be estimated from the electrical resistivity. For $N\left(E_{F}\right)<<1$ the BCS theory predicts (Allen and Mitrov, 1982; Myers, 1997)

$$
T_{c}=1.30 \theta_{D} \exp \left(\frac{1}{N\left(E_{F}\right) V}\right)
$$

This is not a successful way of correlating values of $T_{c}$ for the metallic elements. Nevertheless, it suggests that one should reopen empirically the question of a correlation 
between $T_{c}$ and $\theta_{D}$. There is an interesting apparent paradox: the higher the resistivity at room temperature the more likely it is that a metal will be a superconductor when cooled.

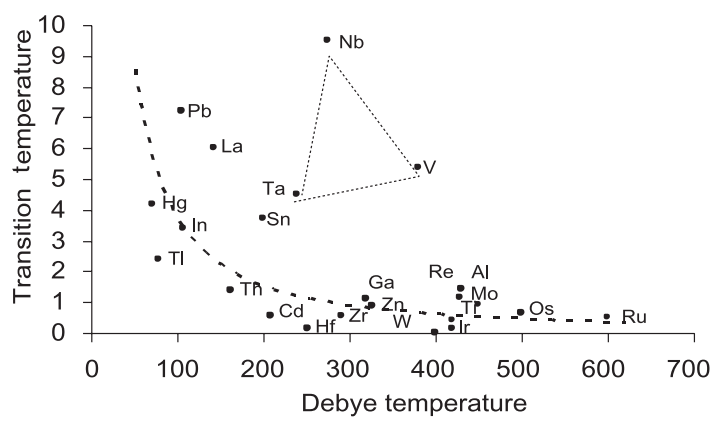

Fig.3(a). Superconducting critical temperatures versus debye temperatures for numerous elements. Redrawn after (Launay and Dolecek, 1947). Solid and dashed lines are guides to the eye. See text for discussion.

De Launay and Dolecek (Launay and Dolecek, 1947) considered the variation of the superconducting transition temperature $T_{c}$ with the Debye characteristic temperature $\theta_{D}$. We have redrawn data by De Launay and Dolecek in fig. 3.

While for mainly non-transition elements the continuous line drawn in fig. 3(a) already given by De Launay and Dolecek (1947) a decade before BCS theory, shows a relation between $T_{c}$ and $\theta_{D}$ is far from simple. And the triangle involving $\mathrm{Ta}, \mathrm{V}$. and $\mathrm{Nb}$ modified from the 1947 figure of De Launay and Dolecek (1947) shows no relation to the continuous curve.

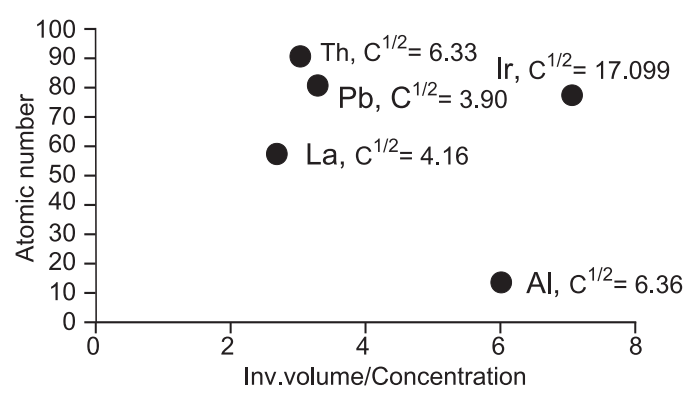

Fig.3(b). Shows five superconducting fcc transition elements in the $(\Omega, Z)$ plane. To each point the values of square root $C^{\frac{1}{2}}$ of the 'average' elastic constant as defined by equation (7) in $\operatorname{GPa}^{\frac{1}{2}}$

Debye temperature $\theta_{D}$ can be related with Cauchy deviation $C^{*}$ by obeying the first principles theory. On the other hand $T_{c}$ is much more related to $C^{*}$ is shown in previous section.

To make a correlation between $T_{c}$ and Cauchy discrepancy, many earlier workers have calculated the Debye temperature for cubic crystals by using the elastic constants $C_{11}, C_{12}$ and $C_{44}$, the mass density $\rho$ and atomic volume $\Omega$. While Houston's method (Houstan, 1948) is favoured and has been developed by (Betts et al. 1956; Betts et al. 1956) we have found the semi-empirical relation of Blackman (Blackman, 1951), quoted in Huntington's review article (Huntington, 1958), an important introductory point which is

$$
\begin{aligned}
& \theta_{D}^{3}=\frac{3.15}{8 \pi}\left(\frac{h}{k_{B}}\right)^{3} \frac{s}{\rho^{\frac{3}{2}} \Omega} \times\left(C_{11}-C_{12}\right)^{\frac{1}{2}}\left(C_{11}+C_{12}+\right. \\
& \left.2 C_{44}\right)^{\frac{1}{2}}\left(C_{44}\right)^{\frac{1}{2}}
\end{aligned}
$$


Here $\mathrm{s}$ is the number of atoms in the unit cell of volume $\Omega$ and $\rho$ is the mass density. Equation (6) can be true into the definition of an 'average' elastic constant, $C$ or $C^{*}$ by approximation.

$$
C^{*}=\left(\frac{8 \pi}{3.15}\right)^{\frac{2}{3}}\left(\frac{k_{B}}{h}\right)^{2} \frac{\rho \Omega^{\frac{2}{3}}}{s^{\frac{2}{3}}} \theta_{D}^{2}
$$

So our final correlation between transition temperature $T_{c}$ and Cauchy discrepancy $C^{*}$ is

$$
T_{c}[K]=A\left(\frac{8 \pi}{3.15}\right)^{\frac{2}{3}}\left(\frac{k_{B}}{h}\right)^{2} \frac{\rho \Omega^{\frac{2}{3}}}{s^{\frac{2}{3}}} \theta_{D}{ }^{2}-B
$$

Fig. 3(b) same as fig. 1, but coordinates in the $(\Omega, \mathrm{Z})$ plane are now labeled by the values of square root $C^{\frac{1}{2}}$ of the average elastic constant. It is clear from this fig. at constant volume $\Omega . C^{\frac{1}{2}}$ related to $\theta_{D}$ through equation (7). Again $C^{\frac{1}{2}}$ increases with increasing $\mathrm{Z}$ in contrast to the behavior of $T_{c}$ in fig. 1 .
At constant $\mathrm{Z}, C^{\frac{1}{2}}$ increases with decreasing atomic volume also. According to BCS theory, we are interested to correlate with but now via the Cauchy discrepancy. In fig. 4 the functional form obtained empirically being recorded in the caption and represent a remarkable correlation for the five fcc elements.

\section{Results and Discussion}

By considering fig. 3, we can draw our conclusion that there is no simple relation between Debye temperature $\theta_{D}$ and the superconducting transition temperature $T_{C}$. Fig. 1 and fig. 3 (b) have been led us to take step to correlate $\mathrm{C}$ having dimension of an elastic constant and defined in equation (7). This average elastic constant can be expressed with $\theta_{D}$ and with the Cauchy discrepancy $C^{*}$ in equation (3). We have focused on these quantities for five fcc elements and got an clear interrelation, as fig. 4 demon

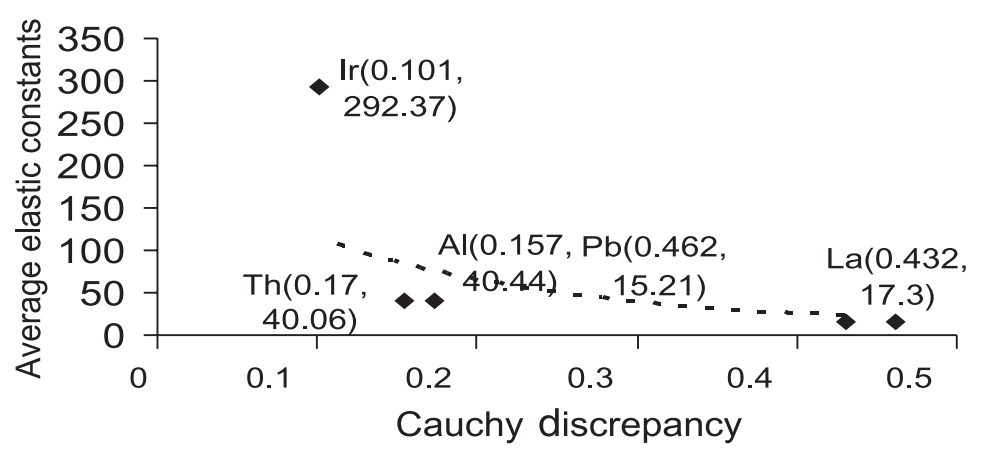

Fig. 4. Shows 'average' elastic constant C, Eq.(7), as a function of Cauchy discrepancy $C^{*}$, $E q .(3)$, for the five fcc transition metals. Dashed line is a guide for the eye, which actually employs an exponential form, of the kind $C=\alpha+\beta \exp \left(-\gamma C^{*}\right)$, with $\alpha=20.5 \mathrm{Gpa}, \beta=$ $300.5 \mathrm{Gpa}, \gamma=10.5 \mathrm{Gpa}$ 
strates and the functional form has been extracted. Since as Ledbetter (1980) already pointed out in $1980, T_{C}$ relates linearly to $C^{*}$ as in fig. 2. Equation (Ledbetter, (1980) made a clear correlation between $T_{C}$ and $\theta_{D}$ with mass density and atomic volume entering through the definition of the average elastic constant. Again we thought the BCS theory in another eye sight for zero temperature quantities which is critical field $H_{C}(0)$ and energy gap $E_{g}(0)$. Here we make a correlation simply with the Cauchy discrepancy for five fcc superconducting transition elements by plotting a graph. discrepancy $C^{*}$. Our main objectives to make a Appendix is to display a remarkable correlation between experimentally estimated values of the critical field $H_{C}(0)$ and the energy gap $E_{g}(0)$, as extracted from tunneling experiments, and the Cauchy discrepancy $C^{*}$ (Eqn.-3). Fig. 5, shows $H_{C}(0)$ plotted against $C^{*}$ for five fcc transition elements on which attention was focused in the body of the text. The data for $H_{C}(0)$ have been taken from Kittel's book (Kittel, 1991; Myers, 1997). Evidently, as for $T_{C}$ in fig. 2, a marked correlation again exists, the dashed line

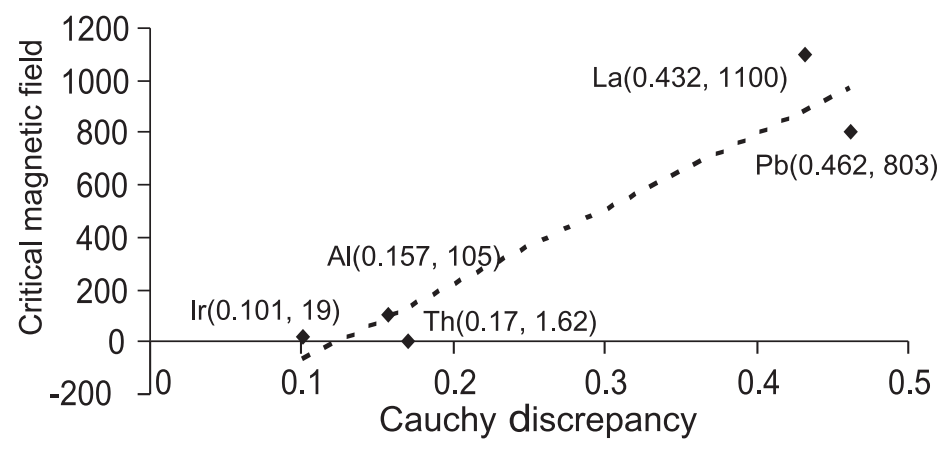

Fig. 5. Experimental results for critical field $H_{C}(0)$, as extracted from tunneling measurements, $V s$ Cauchy discrepancy $C^{*}$, as defined in Equation (3)

Our present studies stimulates advance researcher concerning generalization of basic approach set out here to other group of superconductors.

\section{Appendix}

Here, we have Introduced zero temperature properties that are functions of Cauchy having the equation

$$
H_{C}(0)=b_{1} C^{*}-b_{2}
$$

Here, $b_{1}=0.41$ Tesla and $b_{2}=0.04$ Tesla.

Again we have collected data for the energy gap $E_{g}(0)$ given by Kittel (1991) (see also Poole, et al. 1995), and fig. 6 is shown the same set of five fcc transition elements. The 


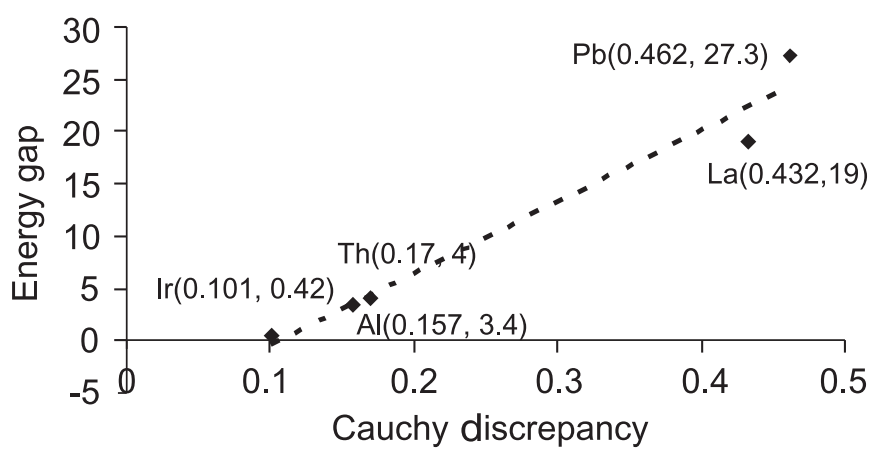

Fig. 6. Experimental results for energy gap $E_{g}(0)$, as extracted from tunneling measurement, $V s$ Cauchy discrepancy $C^{*}$, as defined in Equation (3)

dashed line, but now with more substantial scatter of the experimental points than for either $T_{C}$ or $H_{C}(0)$, have the equation.

$$
E_{g}(0)=\mathrm{e}_{1} \mathrm{C}^{*}-\mathrm{e}_{2}
$$

With $\mathrm{e}_{1}=55.8 \times 10^{-4} \mathrm{eV}$ and $\mathrm{e}_{2}=5.16 \times 10^{-4} \mathrm{eV}$.
BCS theory predicts a constant value for the ratio $E_{g}(0) / K_{B} T_{C}$. On the basis of our present study equation (4) and (10) very nearly fit for the five fcc transition elements. Finally we have plotted the 'average' ratio $E_{g}(0) / K_{B} T_{C}$ from equation (4) and (10) as a function of $C^{*}$ in fig. 7 where using parameter $\mathrm{A}, \mathrm{B}, e_{1}$ and $e_{2}$ are given.

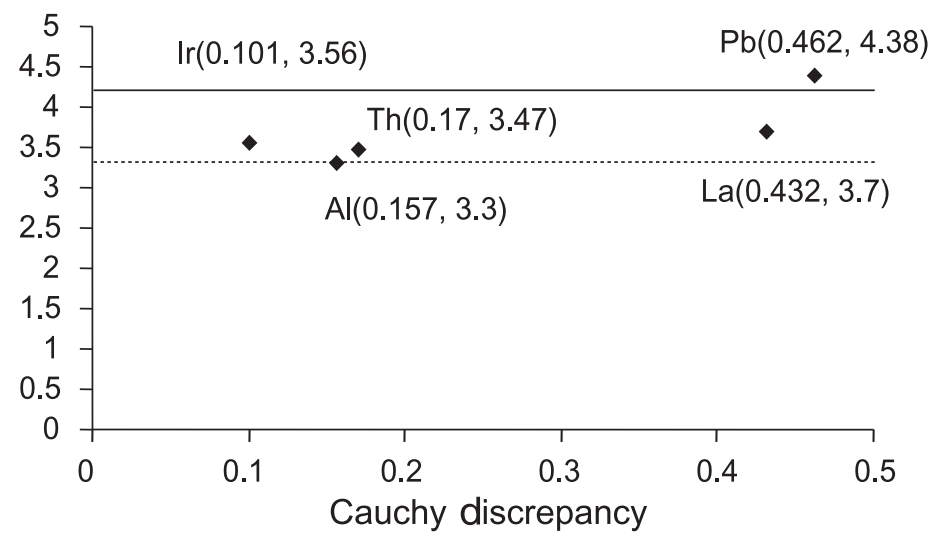

Fig. 7. EBCS ratio $E_{g}(0) / k_{B} T_{c}$ for fcc transition elements. Solid line is ratio of Eqs (4) and (10), While dashed line is BCS theoretical value 


\section{References}

Allen, P.B. and Mitrov, B. (1982) Solid state physics, edited by H. Ehrenreich, F. Seitz, and Turnbull (Academic Press by New york, 1982), 37 : 220-248.

Angilella, G.G.N. Leys, F.E. March, N.H. and Pucci, R. (2004) Correlation between Character-istics energies in non-s-wave Superconductors Phy. Lett. A322, 475-480

Angilella, G.G.N. March, N.H. and Pucci, R. (2000) for non-s-wave pairing Superconductors correlated with coherence length and effective mass. Phys. Rev B 62 : 13919-13921

Angilella, G.G.N. March, N.H. and Pucci, R. (2002) Superconducting Transition Temperatures and coherence length in nons-wave pairing materials correlated with spin fluctuation meditated interaction. Phys. Rev. B 65 : 092509(1-4)

Betts, D.D. Bhatia, A.B. and Horton, G. K. (1956) Debye Characteristic Temperature of Certain Non-cubic Crystals, Phys. Rev.104, 43-47

Betts, D.D. Bhatia, A.B. and Wyman, M. (1956) Houston Method and its application to the calculation of Characteristic Temperature of cubic Crystals, Phys. Rev. 104 : 37-42
Blackman, M. (1951) On the calculation of characteristic temperatures from the Elastic constants, Phil. Mag. 42 : 1441-1442

Hochheimer, H.D. Kuchta, B. Dorhout, P. K. and Yarger, J.L. (Kluwer, Dordrecht, 2001) Schilling, J.S. in frontiers of high pressure research $\mathrm{P}$ : Application of high pressure to low dimensional novel electronic materials, $\mathbf{4 8}$ of NATO science series.

Houstan, W.V. (1948) Normal Vibration of a Crystal Lattice. Rev.Mod. Phys 20 :161-166

Huntington, H.B. (1958) Solid State Physics, edited by F. Seitz and D. Turnbull (Academic Press, New York,), 7 : pp-214-300

Kittel, C. (1991) Introduction to Solid State Physics (J. Wiley and Sons, New York) pp 367, 396

Launay, J. De. and Dolecek, R.L. (1947) Superconductivity and the Debye characteristic temperature, Phys. Rev. $72: 141-143$

Ledbetter, H.M. (1980) Correlation between superconducting transition temperature and the Cauchy discrepancy in a body-centeredcubic transition metals. Phys. Lett. 77 A, 359-361

Mehl, M.J. and Papaconstantopoulos, D.A (1996) Application of a tight-binding total-energy method for transition and noble metals: elastic constant, vacancies, and surfaces of monatomic metals, Phys. Rev. B 54 : 4519 4530. 
Myers, H. P. (1997) Introductory Solid State Physics (Taylor and Francis, London,) pp 182-190.

Poole, Ch.P. Farach, H A. and Creswick, R. J. (1995) Superconductivity (Academic press, San Diego), pp (320-340)
Schrieffer, J.R. (1964) Theory of Superconductivity (W.A .Benjamin, New York) pp (24-57)

Shimizu, K. Kimura, T. Furomoto, S. Takeda, K. Kontani, K. Onuki, Y. and Amaya, K. (2001) Superconductivity in the non-magnetic state of iron under pressure. Nature 412 : 316-318

Received : January 07, 2007;

Accepted : June 04, 2007 